\title{
Susceptibility-Weighted Imaging Findings in Aspartylglucosaminuria
}

\author{
(D) A. Tokola, (1) M. Laine, (D) R. Tikkanen, and (D)T. Autti
}

\begin{abstract}
BACKGROUND AND PURPOSE: Aspartylglucosaminuria is a rare lysosomal storage disorder that causes slowly progressive, childhood-onset intellectual disability and motor deterioration. Previous studies have shown, for example, hypointensity in the thalami in patients with aspartylglucosaminuria on T2WI, especially in the pulvinar nuclei. Susceptibility-weighted imaging is a neuroimaging technique that uses tissue magnetic susceptibility to generate contrast and is able to visualize iron and other mineral deposits in the brain. SWI findings in aspartylglucosaminuria have not been reported previously.
\end{abstract}

MATERIALS AND METHODS: Twenty-one patients with aspartylglucosaminuria (10 girls; 7.4-15.0 years of age) underwent 3T MR imaging. The protocol included an SWI sequence, and the images were visually evaluated. Thirteen patients (6 girls, 7.4-15.0 years of age) had good-quality SWI. Eight patients had motion artifacts and were excluded from the visual analysis. Thirteen healthy children (8 girls, 7.3-14.1 years of age) were imaged as controls.

RESULTS: We found a considerably uniform distribution of decreased signal intensity in SWI in the thalamic nuclei in 13 patients with aspartylglucosaminuria. The most evident hypointensity was found in the pulvinar nuclei. Patchy hypointensities were also found especially in the medial and anterior thalamic nuclei. Moreover, some hypointensity was noted in globi pallidi and substantia nigra in older patients. The filtered-phase images indicated accumulation of paramagnetic compounds in these areas. No abnormal findings were seen in the SWI of the healthy controls.

CONCLUSIONS: SWI indicates accumulation of paramagnetic compounds in the thalamic nuclei in patients with aspartylglucosaminuria. The finding may raise the suspicion of this rare disease in clinical practice.

ABBREVIATIONS: $A G A=$ aspartylglucosaminidase; $\mathrm{AGU}=$ aspartylglucosaminuria; $\mathrm{SI}=$ signal intensity

A spartylglucosaminuria (AGU) is a rare lysosomal storage disorder causing progressive decline in intellectual and motor functions. It is a generalized disease affecting the whole body. The clinical picture was comprehensively described in a previously published review article, ${ }^{1}$ but we have recently also

Received June 12, 2019; accepted after revision September 3.

From the HUS Medical Imaging Center, Radiology (A.T., T.A.), University of Helsinki and Helsinki University Hospital, Helsinki, Finland; Department of Child Neurology (M.L.), Helsinki University Hospital, Helsinki, Finland; and Institute of Biochemistry, Medical Faculty (R.T.), University of Giessen, Giessen, Germany.

This study was supported by the Jane and Aatos Erkko Foundation, the Finnish patient organization Suomen AGU ry, the Rare Trait Hope Fund (R.T.), the Scientific Fund of HUS Medical Imaging Center of Helsinki University Hospital (A.T.), the Pediatric Research Center (M.L.) and the Lastenlinna Foundation (M.L.)

Paper previously presented, in part, at: Annual Meeting of European Society of Neuroradiologists, September 19-23, 2018; Rotterdam, the Netherlands.

Please address correspondence to Anna Tokola, HUS Medical Imaging Center, PO Box 340, 00029 HUS, Finland; e-mail: anna.tokola@hus.fi

Indicates article with supplemental on-line table.

http://dx.doi.org/10.3174/ajnr.A6288 published new data on cognitive profiles of school-aged patients with AGU. ${ }^{2}$ Children with AGU appear healthy at birth. An infantile growth spurt and respiratory infections and hernias are among the first signs of the disease. Delayed speech development and clumsiness are noted in early childhood. Typical facial features including a short and broad nose with periorbital fullness developing during the first years. ${ }^{3}$ An abnormally slow-but-positive intellectual and motor development up to teenage years is typical. After a relatively stable period of about 10 years up to 15 years of age, there is first a slow decline in intellectual and motor functions up to the age of 25-28 years, followed by a more rapid decline, finally resulting in severe mental impairment. ${ }^{2}$ There are notable individual differences, but the disease leads to death usually before 50 years of age.

AGU is an inherited, autosomal recessive disorder caused by a mutation in the aspartylglucosaminidase ( $A G A$ ) gene located on 4q34.3. ${ }^{4}$ The disease is enriched in the Finnish population, and about $98 \%$ of the Finnish patients with AGU are homozygous for a specific point mutation, AGUFIN major. ${ }^{5}$ The data from the 
Human Gene Mutation Database lists $>30$ different AGU disease-causing mutations in the $A G A$ gene. ${ }^{6}$ In Finland, the prevalence is approximately 1.7-5/100,000 live births. Sporadic cases have been reported globally, but the worldwide incidence is unknown.

The AGUFIN major mutation causes local misfolding and deficient activity of the aspartylglucosaminidase enzyme, which catalyzes the hydrolysis of aspartylglucosamine and other glycoasparagines. ${ }^{7}$ This results in accumulation of undegraded aspartylglucosamine and other glycoasparagines in body fluids and tissues of patients with AGU. Hypertrophied storage lysosomes are present in all tissues and organs.

Therapies for many lysosomal storage disorders have improved during the past decades, but no approved treatment is currently available to cure or slow down the progression of AGU. Recently, a chaperone treatment trial in patients with AGU was initiated, and the patients in our study are currently participating in the trial. The treatment exploits a small chemical compound that functions as a pharmacologic chaperone for the mutated $A G A .^{8}$ Small molecules known as pharmacologic chaperones bind to their protein targets and stabilize their native structures, helping mutated proteins to regain their biologic function. In preclinical studies, treatment of patient fibroblasts with these compounds resulted in increased $A G A$ activity and improved lysosomal morphology in patient fibroblasts with 2 different AGU mutations. ${ }^{8}$

Previously, mouse-model studies investigating virus-mediated gene therapy and enzyme replacement therapy in AGU have shown some metabolic correction and decreased storage in the brain. ${ }^{9,10}$ So far, gene therapy or enzyme replacement therapy trials have not been reported in human patients with AGU. Bone marrow transplantation was investigated on a small group of patients with AGU, with no long-term benefit. ${ }^{11}$

Previous MR imaging studies in patients with AGU have shown, for example, a T2 signal intensity decrease in the thalami and cerebral and/or cerebellar atrophy at later stages of the disease. SWI is a velocity-corrected gradient-echo MR imaging sequence with a high spatial resolution. It uses tissue magnetic susceptibility differences to generate contrast, ${ }^{12,13}$ providing information about tissues that have a different susceptibility than the surrounding tissues. The susceptibility difference can be caused by substances such as deoxygenated blood, hemosiderin, ferritin, and calcium. Filtered-phase images can be used to differentiate between paramagnetic substances (ie, iron) and diamagnetic substances (calcium). ${ }^{14}$ The paramagnetic and diamagnetic substances have opposite signal intensities on filtered-phase images, but the particular signal intensity depends on the scanner and the manufacturer. If the scanner is so-called "left-handed," the paramagnetic substances appear bright in the filtered-phase images, whereas if the scanner is "right-handed," the paramagnetic substances appear dark.

SWI has several clinical applications in the neuroimaging field. The method has been used to monitor the amount of iron in the brain in various diseases and conditions, including aging, Alzheimer disease, stroke, trauma, tumors, and multiple sclerosis. ${ }^{13,15,16}$ To our knowledge, SWI findings in AGU have not been previously described in the literature, and our aim was to evaluate whether SWI is able to depict mineral deposits and their distribution in patients with AGU.

\section{MATERIALS AND METHODS}

We imaged 21 patients (10 girls, 7.4-15.0 years of age; mean, $10.9 \pm 2.4$ years) and 13 healthy controls (8 girls, 7.3-14.1 years of age; mean, $10.2 \pm 2.3$ years) with MR imaging (3T Magnetom Skyra; Siemens, Erlangen, Germany). The patients are participants of a recently initiated chaperone treatment trial, and they were imaged before the onset of treatment. The protocol included the following sequences: T1-weighted 3D-MPRAGE (TR $=2000$ $\mathrm{ms}, \mathrm{TE}=2.74 \mathrm{~ms}$, flip angle $=10^{\circ}$, thickness $=1 \mathrm{~mm}$, matrix $=$ $256 \times 256)$, FLAIR 3D $(\mathrm{TR}=5000 \mathrm{~ms}, \mathrm{TE}=386 \mathrm{~ms}, \mathrm{TI}=1800$ $\mathrm{ms}$, flip angle $=120^{\circ}$, thickness $=1 \mathrm{~mm}$, matrix $\left.=244 \times 244\right)$, T2weighted TSE axial $\left(\mathrm{TR}=4000 \mathrm{~ms}, \mathrm{TE}=82 \mathrm{~ms}\right.$, flip angle $=150^{\circ}$, thickness $=3 \mathrm{~mm}$, matrix $=448 \times 448)$, diffusion-weighted imaging $\left(\mathrm{TR}=10160 \mathrm{~ms}, \mathrm{TE}=64 \mathrm{~ms}\right.$, flip angle $=180^{\circ}$, thickness $=$ $3 \mathrm{~mm}$, matrix $=160 \times 160)$, diffusion tensor imaging $(\mathrm{TR}=3700$ $\mathrm{ms}, \mathrm{TE}=108 \mathrm{~ms}$, flip angle $=90^{\circ}$, thickness $=2.4 \mathrm{~mm}$, matrix $=$ $98 \times 96)$, and susceptibility-weighted imaging $(\mathrm{TR}=27 \mathrm{~ms}, \mathrm{TE}=$ $20 \mathrm{~ms}$, flip angle $=15^{\circ}$, thickness $=2 \mathrm{~mm}$, matrix $=256 \times 232$ ). The scanner manufacturer uses a so-called "left-handed" reference scheme in the SWI filtered-phase images.

Twelve patients were sedated for the MR imaging examination, whereas 9 patients were imaged without sedation. Eight of these unsedated patients had motion artifacts and were excluded from the SWI analysis, so images of altogether 13 patients ( 6 girls, 7.4-15.0 year of age; mean, $11.2 \pm 2.5$ years) were analyzed by 2 radiologists (A.T. and T.A.).

The study was approved by the local ethical committee. Written informed consent was obtained from the parents of the participants because the participants were minors and intellectually disabled.

\section{RESULTS}

Interestingly, all 13 patients with good-quality SWI showed a highly similar distribution of hypointensity in the thalamic nuclei (Fig 1). The most evident hypointensity was found in the pulvinar nuclei. Patchy hypointensities were also found, especially in the medial and anterior nuclei. Moreover, some hypointensity was noted in the globi pallidi in 6 of the patients, especially in the medial aspect. In 5 patients between 11.5 and 15.0 years of age, hypointensity was also evident in the substantia nigra. The filtered-phase images indicated accumulation of paramagnetic compounds in these areas (Fig 2). No microhemorrhages, lobar hemorrhages, or signs of subarachnoid hemorrhage were seen.

In T2WI, a signal intensity (SI) decrease was seen in all patients in the thalami, with a more intense decrease in the pulvinar nuclei, which is a previously described typical finding in AGU. ${ }^{17}$ The SI in the thalami was normal on T1WI in all patients. Mild T2 hyperintensity in the WM was found in all patients, especially in the periventricular and deep white matter. A more intense thin lining of periventricular hyperintensity was seen in the FLAIR images in nearly all patients. Deficiencies of differentiation between gray and white matter 
in the frontal and temporal lobes were common findings. Mild atrophy in the form of a thin corpus callosum, mildly dilated ventricles or cortical sulci, or cerebellar atrophy was also common in the patient group. The findings in $\mathrm{T} 1$ - and

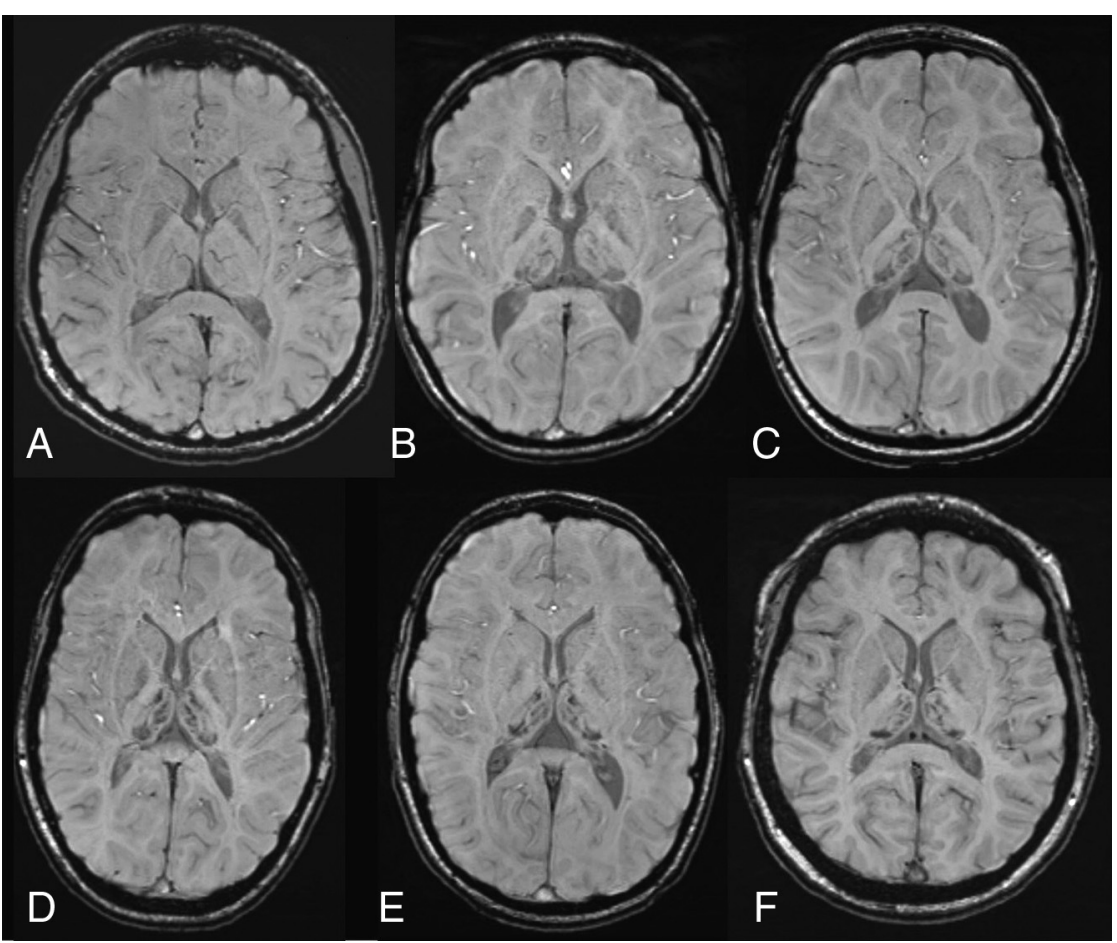

FIG 1. Susceptibility-weighted axial images at the level of basal ganglia and thalami. $A, \mathrm{~A}$ healthy control, an 11-year-old girl with normal findings. B-F, Five patients with AGU at different ages (7.9, 9.5, 11.4, 13.8, and 14.2 years) show a strikingly similar distribution of hypointensity in deep gray matter structures, with a maximum-intensity decrease in the pulvinar nuclei. Patchy decrease is also noted in the medial and anterior thalamic nuclei. Some signal intensity decrease is also seen in the globi pallidi.
T2-weighted sequences correspond to previously reported typical MR imaging findings in AGU. ${ }^{17,18}$ The MR imaging findings are described in the On-line Table, and images of one patient are presented in Fig 3.

In those patients with AGU who were excluded from the SWI visual analysis due to motion artifacts, it was possible to see some SI decrease in SWI in the thalami, but more precise evaluation was not possible. In the healthy control group, no abnormal SI decrease was seen in the deep gray matter, and no other clinically relevant findings were noted in SWI or other sequences.

\section{DISCUSSION}

Histopathology, Cell Biology, and Role of Iron in AGU and Some Other Lysosomal Storage Disorders

In a study investigating the cerebral histopathology in patients with AGU, the basic cortical cytoarchitecture was generally preserved, but most neurons contained vacuoles that were also found in the neurons of the deep gray matter, including the thalami. In 2 of the 4 postmortem cases, the white matter showed a diffuse pallor of myelin staining and some gliosis. ${ }^{19}$ Mouse model studies have shown similar histopathologic findings. ${ }^{20}$

The identity of the paramagnetic compounds causing the SWI hypointensity in the thalamic nuclei in patients

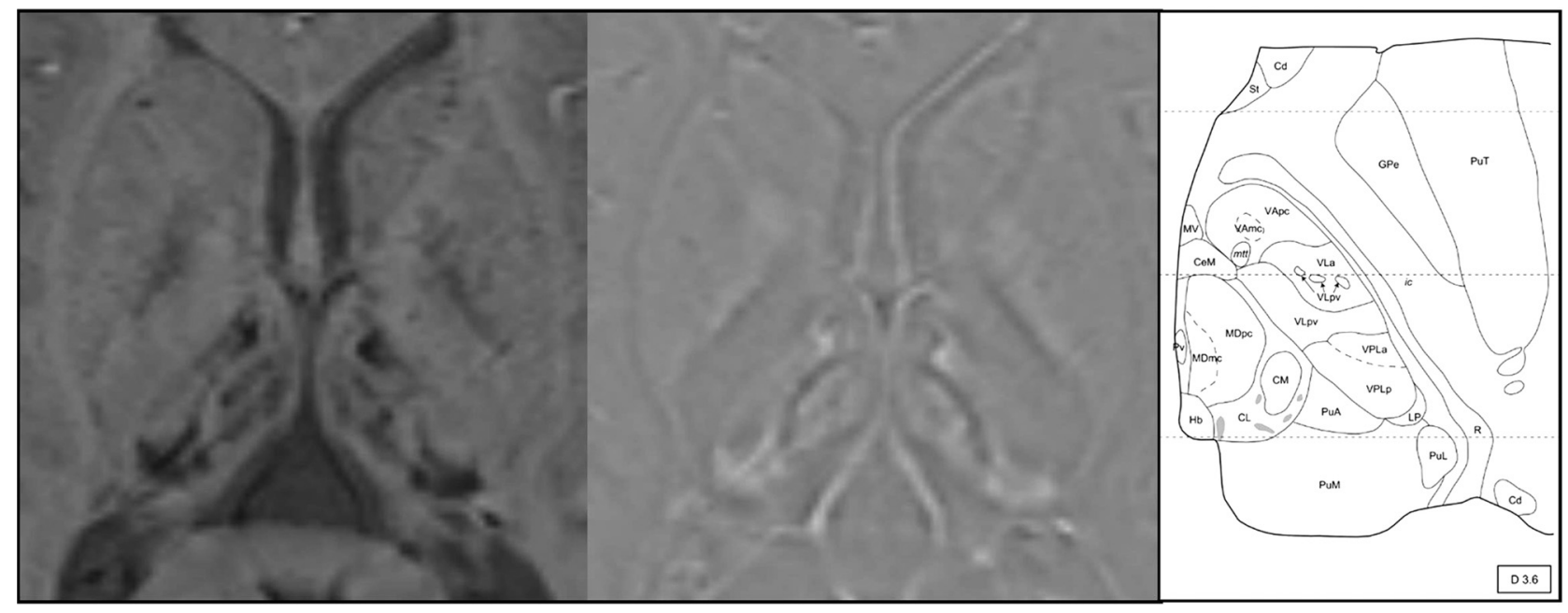

FIG 2. On the left, susceptibility-weighted axial image and magnification of the thalami in a 13-year-old boy with AGU showing the distribution of decreased signal intensity in the thalamic nuclei. The most evident decrease is seen in the pulvinar nuclei, but a patchy signal intensity decrease is also seen in the medial and anterior nuclei. In the middle, a filtered-phase image indicates accumulation of paramagnetic compounds in these areas. On the right, a schematic illustration of the nuclei of the left thalamus (figure from the atlas by Morel used with permission ${ }^{29}$ ). Copyright @ 2019 From Stereotactic Atlas of the Human Thalamus and Basal Ganglia by Anne Morel. Reproduced by permission of Taylor and Francis Group, LLC, a division of Informa plc. 


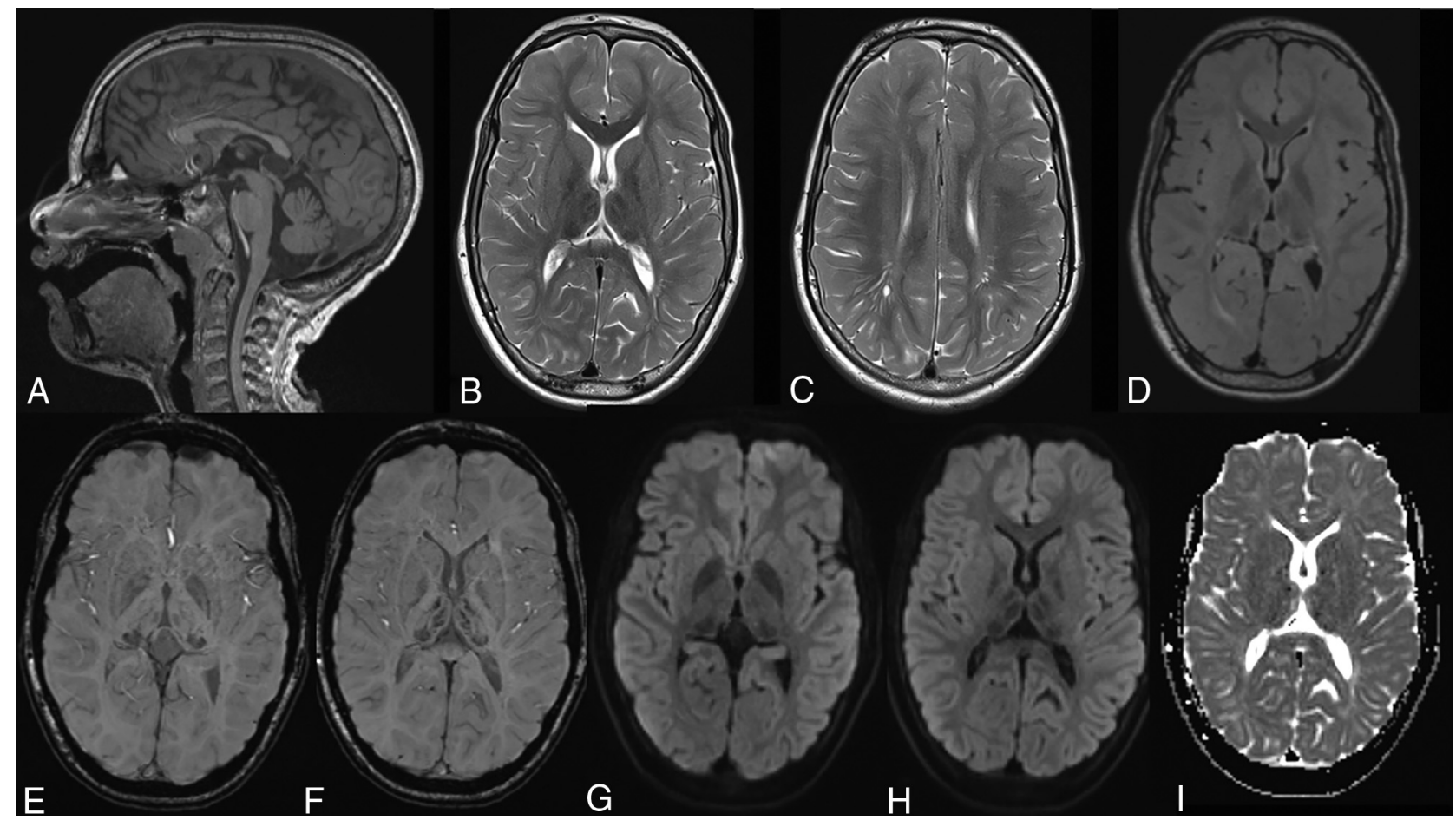

FIG 3. Typical MR imaging findings in an 11-year-old girl with AGU. A, Sagittal TTWI shows a relatively thin corpus callosum and a pineal cyst. $B$, Axial T2WI with typical hypointensity in the thalami and especially in the pulvinar nuclei. C, Axial T2WI shows hyperintensity in the white matter, poor differentiation between gray and white matter especially in frontal lobe, and some mildly dilated perivascular spaces. D, Axial FLAIR image also shows the hypointensity in the pulvinar nuclei and hyperintensity in the white matter. $E$ and $F$, Axial SWI with hypointensity in the pulvinar and medial and anterior nuclei in the thalami. G-I, Axial DWI and ADC images show no signs of restricted diffusion.

with AGU remains unclear. For example, free radicals, iron, manganese ion, or deoxyhemoglobin, each of which contain unpaired electrons, may cause such an effect. On the basis of studies of cell biology and iron deposition mechanisms in other lysosomal storage disorders, it may be assumed that the finding in SWI is caused by iron in some form.

There are potential explanations for iron deposition seen especially in the thalami and other deep gray matter structures. In the brain, most of the iron is found in the oligodendrocytes and their processes, and there is a high oligodendrocyte density in the deep gray matter. ${ }^{21}$ The SWI SI decrease may also be a result of iron accumulation of the microglia. It has been proposed that neuroinflammation is a factor in many lysosomal storage disorders, and it may negatively impact neuronal survival and contribute to neurodegeneration. ${ }^{22}$ Microglia and astrocyte activation and influx of highly iron-laden microglia in the thalami and other structures are also possible mechanisms for iron deposition visualized by SWI. In lysosomal storage disorders, substrate accumulation disrupts the normal homeostatic function, including iron homeostasis. The accumulation of iron and its toxic effects, related to the increased cellular oxidative stress, may also have an important role in the downstream cellular pathways, resulting in cell and tissue dysfunction. ${ }^{23,24}$

In $\mathrm{AGU}$, as the disease progresses, there are widespread changes in the brain parenchyma, for example, delayed or deficient myelination and, at later stages, also demyelination, neuronal loss, and atrophy. It is, therefore, not possible to evaluate the role of thalamic iron deposition and possible thalamic dysfunction in the various neuropsychiatric symptoms. However, thalamic pathology has been shown to correlate with cognitive dysfunction, and it may also play a role in the clinical picture of AGU. The functions of the thalamus include relaying sensory signals as well as motor signals to the cerebral cortex, but also regulation of consciousness, sleep, and alertness. Thalamocortical connectivity is associated with diverse functions of higher level cognitive processes, such as working and episodic memory, attention, and speed of information processing, ${ }^{21,25}$ which are impaired in AGU as the disease progresses.

In a previous report of adolescent twins, one of whom had AGU, the volume of the thalami was about $30 \%$ smaller in the twin with AGU than in the healthy twin brother, ${ }^{26}$ and the difference in volume progressed during the 4-year follow-up. This may be due to demyelination and neuronal loss. The observed SI decrease in the T2WI and SWI seen in the present study might, in part, be due to condensation of iron in the tissue, but there is apparently also an increased amount of iron due to disturbed function of lysosomes and disrupted iron homeostasis.

Iron deposition in the brain is a common finding in elderly individuals, but in healthy children and adolescents, the deep GM nuclei do not show signs of iron or other mineral deposits on SWI. In our study, the SI decrease in the pulvinar nuclei was noted even in the youngest participants with AGU, who were 7 years of age. It is possible that this finding could be observed even in younger children with AGU.

\section{Limitations and Future Aspects}

The limitation of the study is the relatively small number of patients, which is due to the rarity of the disease. In the future, it will be interesting to see whether the emerging treatments have 
an effect on the MR imaging findings in AGU, including the deposition of paramagnetic compounds seen in SWI. Quantitative susceptibility mapping, which is a novel technique for the assessment of magnetic tissue susceptibility differences, ${ }^{27,28}$ might also be used to determine disease-related iron concentration in AGU.

\section{CONCLUSIONS}

SWI showed a recognizable distribution of SI decrease in the thalamic nuclei in children and adolescents with AGU, and some of the patients also had an SI decrease in the globi pallidi and substantia nigra. The described pattern, together with other previously described MR imaging findings, may raise the suspicion of this rare disease when observed in patients with an unknown diagnosis in clinical practice. This will be increasingly important when, hopefully, there is an efficient treatment available for this disease in the near future.

\section{ACKNOWLEDGMENTS}

The authors would like to thank the participants and their families and also the Finnish patient organization Suomen AGU ry for their valuable help with the recruitment.

Disclosures: Anna Tokola—RELATED: Grant: Scientific Fund of HUS Medical Imaging Center of Helsinki University Hospital, Comments: Grant was used for writing this article. Minna Laine-RELATED: Grant: Jane and Aatos Erkko Foundation, Lastenlinna Foundation, Pediatric Research Center; UNRELATED: Travel/Accommodations/Meeting Expenses Unrelated to Activities Listed: Shire/Takeda, Orion, Taylor's Tale. Ritva Tikkanen-RELATED: Grant: Jane and Aatos Erkko Foundation, Comments: grant for research; Support for Travel to Meetings for the Study or Other Purposes: Suomen AGU ry, Comments: travel grants for meetings due to the study; UNRELATED: Grants/Grants Pending: Deutsche Forschungsgesellschaft, Succinic Semialdehyde Dehydrogenase Deficiency Association, NCL-Stiftung, Comments: all research grants.

\section{REFERENCES}

1. Arvio M, Mononen I. Aspartylglycosaminuria: a review. Orphanet $J$ Rare Dis 2016;11:162 CrossRef Medline

2. Harjunen E, Laine M, Tikkanen R, et al. Detailed profile of cognitive dysfunction in children with aspartylglucosaminuria. J Inherit Metab Dis 2019 Aug 15. [Epub ahead of print] CrossRef Medline

3. Arvio $\mathrm{P}$, Arvio M. Progressive nature of aspartylglucosaminuria. Acta Paediatr 2002;91:255-57 CrossRef Medline

4. Saarela J, Laine M, Oinonen C, et al. Molecular pathogenesis of a disease: structural consequences of aspartylglucosaminuria mutations. Hum Mol Genet 2001;10:983-95 CrossRef Medline

5. Syvänen AC, Ikonen E, Manninen T, et al. Convenient and quantitative determination of the frequency of a mutant allele using solidphase minisequencing: application to aspartylglucosaminuria in Finland. Genomics 1992;12:590-95 CrossRef Medline

6. Stenson PD, Mort M, Ball EV, et al. The Human Gene Mutation Database: building a comprehensive mutation repository for clinical and molecular genetics, diagnostic testing and personalized genomic medicine. Hum Genet 2014;133:1-9 CrossRef Medline

7. Mononen I, Fisher KJ, Kaartinen V, et al. Aspartylglycosaminuria: protein chemistry and molecular biology of the most common lysosomal storage disorder of glycoprotein degradation. FASEB $J$ 1993;7:1247-56 CrossRef Medline

8. Banning A, Gülec C, Rouvinen J, et al. Identification of small molecule compounds for pharmacological chaperone therapy of aspartylglucosaminuria. Sci Rep 2016;6:37583 CrossRef Medline
9. Dunder U, Valtonen P, Kelo E, et al. Early initiation of enzyme replacement therapy improves metabolic correction in the brain tissue of aspartylglycosaminuria mice. J Inherit Metab Dis 2010;33:611-17 CrossRef Medline

10. Virta S, Rapola J, Jalanko A, et al. Use of nonviral promoters in adenovirus-mediated gene therapy: reduction of lysosomal storage in the aspartylglucosaminuria mouse. J Gene Med 2006;8:699-706 CrossRef Medline

11. Arvio M, Sauna-Aho O, Peippo M. Bone marrow transplantation for aspartylglucosaminuria: follow-up study of transplanted and non-transplanted patients. J Pediatr 2001;138:288-90 CrossRef Medline

12. Haacke EM, Xu Y, Cheng Y-C, et al. Susceptibility weighted imaging (SWI). Magn Reson Med 2004;52:612-18 CrossRef Medline

13. Haacke EM, Mittal S, Wu Z, et al. Susceptibility-weighted imaging: technical aspects and clinical applications, Part 1. AJNR Am J Neuroradiol 2009;30:19-30 CrossRef Medline

14. Schweser F, Deistung A, Lehr BW, et al. Differentiation between diamagnetic and paramagnetic cerebral lesions based on magnetic susceptibility mapping. Med Phys 2010;37:5165-78 CrossRef Medline

15. Ropele S, Enzinger C, Fazekas F. Iron mapping in multiple sclerosis. Neuroimaging Clin N Am 2017;27:335-42 CrossRef Medline

16. Sehgal V, Delproposto Z, Haacke EM, et al. Clinical applications of neuroimaging with susceptibility-weighted imaging. J Magn Reson Imaging 2005;22:439-50 CrossRef Medline

17. Tokola AM, Åberg LE, Autti TH. Brain MRI findings in aspartylglucosaminuria. J Neuroradiol 2015;42:345-57 CrossRef Medline

18. Autti T, Lonnqvist T, Joensuu R. Bilateral pulvinar signal intensity decrease on T2-weighted images in patients with aspartylglucosaminuria. Acta Radiol 2008;49:687-92 CrossRef Medline

19. Autti T, Raininko R, Haltia M, et al. Aspartylglucosaminuria: radiologic course of the disease with histopathologic correlation. J Child Neurol 1997;12:36 CrossRef Medline

20. Jalanko A, Tenhunen K, McKinney CE, et al. Mice with an aspartylglucosaminuria mutation similar to humans replicate the pathophysiology in patients. Hum Mol Genet 1998;7:265-72 CrossRef Medline

21. Schweser F, Raffaini Duarte Martins AL, Hagemeier J, et al. Mapping of thalamic magnetic susceptibility in multiple sclerosis indicates decreasing iron with disease duration: a proposed mechanistic relationship between inflammation and oligodendrocyte vitality. Neuroimage 2018;167:438-52 CrossRef Medline

22. Bosch ME, Kielian T. Neuroinflammatory paradigms in lysosomal storage diseases. Front Neurosci 2015;9:417 CrossRef Medline

23. Futerman AH, Van Meer G. The cell biology of lysosomal storage disorders. Nat Rev Mol Cell Biol 2004;5:554-65 CrossRef Medline

24. Bras JM. Lysosomal storage disorders and iron. Int Rev Neurobiol 2013;110:251-75 CrossRef Medline

25. Mitchell AS, Sherman SM, Sommer MA, et al. Advances in understanding mechanisms of thalamic relays in cognition and behavior. J Neurosci 2014;34:15340-46 CrossRef Medline

26. Tokola A, Brandstack N, Hakkarainen A, et al. Erratum: White matter microstructure and subcortical gray matter structure volumes in aspartylglucosaminuria; a 5-year follow-up brain MRI study of an adolescent with aspartylglucosaminuria and his healthy twin brother. JIMD Rep 2017;35:117 CrossRef Medline

27. Wang Y, Liu T. Quantitative susceptibility mapping (QSM): decoding MRI data for a tissue magnetic biomarker. Magn Reson Med 2015;73:82-101 CrossRef Medline

28. Schweser F, Deistung A, Lehr BW, et al. Quantitative imaging of intrinsic magnetic tissue properties using MRI signal phase: an approach to in vivo brain iron metabolism? Neuroimage 2011;54:2789-807 CrossRef Medline

29. Morel A. Stereotactic Atlas of the Human Thalamus and Basal Ganglia. Boca Raton; CRC Press; 2007 